Clinical evaluation consisted in the evaluation of the NAPSI and PASI scores. The MSUS evaluation consisted in the evaluation of 10 hand nails. In B-mode (BM) we evaluated the followings: thickness of the nail bed from the distal phalanx bone surface to the ventral plate (PB) according to Worstman $\mathrm{X}$ et al.; thickness of the nail from dorsal to ventral plate (IP); dorsal and ventral plate morphology, echogenicity and integrity. Additionally, we performed a color Doppler (CD) evaluation for the presence of $C D$ signal at the nail bed and matrix level. A score for BM and different scores for CD were calculated for each nail and sums of all nails for $\mathrm{BM}$ and $\mathrm{CD}$ scores were calculated for each patient.

Results: We evaluated 60 patients with PsA, 23 with Pso and 20 controls. $52.4 \%$ were female. The mean age (SD; range) was $50.2(13.6 ; 23-83)$. The age was higher in patients (Pso and PsA) than in controls $(p<0.001)$. Patients with PsA were more treated with DMARD $(81.7 \%)$ while patients with Pso were more treated with topics $(73.9 \%)$ than DMARDs $(13 \%),(p<0.001)$. The majority of the patients $(96 \%)$ had a PASI score less than 12 . The NAPSI was higher in Pso patients than in PsA patients $(p<0.001)$; for all controls the NAPSI was 0 .

US measurements of IP and PB were significantly higher in patients than in controls in the majority of the nail $(p<0.045)$. Total US score for BM was significantly higher in patients than in controls $(p<0.001)$. There were no significant differences for the majority of $C D$ scores between patients and controls.

Overall we found weak to moderate positive correlations between NAPSI and US scores for BM, both for matrix and bed. For most of the nails we found no correlation between NAPSI and CDUS scores; for the rest of the nails the correlation was weak, both positive and negative.

References:

[1] The MSUS measurements and scores showed to be higher in patients with PsA and Pso compared with controls, while CD scores showed no differences. Disclosure of Interest: None declared

DOI: 10.1136/annrheumdis-2017-eular.4126

\section{AB1030 IDENTIFICATION OF VERTEBRAL FRACTURES IN FRACTURE LIASION SERVICES ACROSS THE UNITED KINGDOM}

J. Sayer, S. Stephenson. Service Development, National Osteoporosis Society, Bath, United Kingdom

Background: Fracture Liaison Services (FLS) can prevent secondary fracture through systematic identification of low trauma fractures using dedicated case finding, with assessment and treatment of osteoporosis where necessary. Services are now being measured for quality against Clinical Standards for Fracture Liaison Services published by the National Osteoporosis Society in 2015.[1] The first standard asserts that all patients over 50 years with a newly reported vertebral fracture will be systematically and proactively identified.

Objectives: To evaluate provision for systematic identification of newly reported vertebral fractures in patients aged over 50 at Fracture Liaison Services (FLS) across the UK.

Methods: A gap analysis tool was used to measure service provision against standard one of the Clinical Standards for Fracture Liaison Services, relating to the systematic and proactive identification of vertebral fractures. Data was collected at 78 sites in the UK.

Results: $63 \%$ (49) of sites had no systematic process in place to identify vertebral fractures. Only $10 \%$ (8) sites identified all newly reported vertebral fractures. $27 \%$ (21) had procedures in place to identify some vertebral fractures, i.e. those within certain cohorts. There was considerable disparity across the UK. Sites in Scotland were significantly more likely to have comprehensive processes in place $(38 \%, 6 / 16)$ than in the rest of the UK $(3 \%, 2 / 62)$.

Conclusions: Systematic identification of vertebral fractures poses a particular challenge to services due to a number of factors. Vertebral fractures are difficult to identify as they tend not to present or be admitted in acute settings where FLS are primarily based. In addition, services require support from Radiology, including a commitment to avoid ambiguous terminology when reporting vertebral fractures. Furthermore, as a category, vertebral fractures fall between departments (Rheumatology, Orthopaedic, Fracture Clinic, A\&E, Spinal services) making systematic identification even more challenging. In the Fracture Liaison Service Database Facilities Audit (May 2016), the most frequently cited barrier to the identification of vertebral fractures was lack of a patient pathway.[2] Gap analysis shows a paucity of provision in the identification of vertebral fractures. This is the key driver for work underway in the NOS to develop a patient pathway for vertebral fractures, in conjunction with clinical experts, to promote best practice and best patient care.

References:

[1] https://www.nos.org.uk/health-professionals/fracture-liaison-services.

[2] https://www.rcplondon.ac.uk/projects/outputs/fls-db-facilities-audit-flsbreakpoint-opportunities-improving-patient-care.

Disclosure of Interest: None declared

DOI: 10.1136/annrheumdis-2017-eular.3464

\section{AB1031 ANTI-DRUG ANTIBODIES: ASSAY PERFORMANCE IN PATIENTS TREATED WITH ANTI-TNF BIODRUGS}

B. Hock ${ }^{1}$, J.L. O'donnell ${ }^{2}$, J. Liu ${ }^{2}$, P. Keating ${ }^{2}$, M. Spellerberg ${ }^{2}$, L. Stamp ${ }^{3}$, M. Barclay ${ }^{3} .{ }^{1}$ Haematolgy Research Group, University of Otago; ${ }^{2}$ Immunology Section, Canterbury Health Labs; ${ }^{3}$ Medicine, University of Otago, Christchurch, New Zealand

Background: Minimum biodrug concentrations of $\sim 7 \mathrm{mg} / \mathrm{l}$ are predictive of disease remission ${ }^{1}$. Very low/absent biodrug concentrations associate with loss of benefit which may be due to $A D A^{2}$ however the clinical utility of $A D A$ is assay dependant. In rheumatoid arthritis the combination of low/absent drug concentration and the presence of ADA appears to have the greatest utility ${ }^{3}$. Canterbury Health Laboratories, New Zealand (CHL) has developed a competitive binding ELISA to detect neutralising antibodies whereas most commercial assays utilise a bridging methodology

Objectives: Compare performance of a competitive binding assay with two commercial bridging assays in the detection of ADA to anti-TNF $\alpha$ biodrugs in serum samples with low/absent biodrug concentration

Methods: Serum samples referred for anti TNF biodrug concentrations found to have very low/undetectable concentrations $(<1 \mathrm{mg} / \mathrm{l})$ were tested for ADA using the competitive-bind assay and two bridging assays (TANI Medikal and GRIFOLS) Results: Over a 22 month period (Jan 2014 - Oct 2016), 67\% (331/497) of referred samples had biodrug concentrations below $7 \mathrm{mg} / \mathrm{l}$ and $15 \%(\mathrm{n}=79)$ had low/undetectable biodrug concentrations (adalimumab $n=36$ or infliximab $n=43$ ). ADAs were detected in 53\% (42/79) of this latter group. The competitive binding assay detected ADAs in all samples testing positive for ADA by binding assay. In addition a further 8 samples were positive for ADA by the competitive assay: $53 \%(42 / 79)$ positive for ADA by the competitive assay and 33\% positive by one or other of the commercial assays

Conclusions: The competitive binding ELISA was more sensitive in detecting biodrug ADAs in serum samples with very low/undetectable biodrug concentrations References:

[1] Felice C, Marco M, Pugliese D,et al. Expert Opin Biol Ther 2015;15:1107-1117.

[2] Schaeverbeke T, Truchetet M, Kostine M, Barnetche T et al. Rheumatology 2016:55:210-220.

[3] Jani M,Chinoy, Warren R, et al. Arthritis and Rheumatology 2015;67:20112019.

Disclosure of Interest: None declared

DOI: 10.1136/annrheumdis-2017-eular. 1757

\section{AB1032 COMPARISON OF DIFFERENT TECHNIQUES FOR DETECTING ANTI-DFS70 ANTIBODIES}

J.M. López-Ortega ${ }^{1}$, Á. Sánchez-Herrero ${ }^{1}$, N. Estañ-Capell ${ }^{1}$, D. YbáñezGarcía $^{2}$, E. Valls-Pascual ${ }^{2}$, C. Vergara-Dangond ${ }^{2}$, M. Aguilar-Zamora ${ }^{2}$ L. Montolio-Chiva ${ }^{2}$, À. Martínez-Ferrer ${ }^{2}$, J.J. Alegre-Sancho ${ }^{2} .{ }^{1}$ Laboratory; ${ }^{2}$ Rheumatology, Hospital Universitario Doctor Peset, Valencia, Spain

Background: Antinuclear antibodies (ANA) positivity with a dense fine speckled (DFS) pattern by indirect immunofluorescence (IIF), as per the definition of the International Consensus on ANA Patterns (ICAP), is not uncommon and is linked to the presence of anti-DFS70 antibodies. These antibodies, in the absence of others, are very valuable as biomarkers of exclusion of a systemic autoimmune disease (SAD).

Objectives: To evaluate anti-DFS70 antibody detection by two different laboratory techniques and its relation with different IIF patterns, including DFS pattern.

Methods: During three months, the serum of patients with positive ANA was consecutively collected. Three groups of patients were established according to their IIF pattern: a first group (D) with a DFS pattern as per ICAP; a second group (M) with other speckled with positive mitosis patterns; and a third group (C), as a control, with well defined homgeneous and speckled patterns.

In order to perform a preliminary analysis, 10 serum samples were randomly selected from each group. In each serum sample, an ANA screening by IIF on Hep-2000 cells (Fluorescent lgG ANA-Ro Test System - Immunoconcepts) using an AP-16 Elite/Zenit-Up/GSight system from Menarini, and an anti-DFS70 antibodies detection by two different laboratory techniques (IIF on Hep-2 cells [Hep-2/DFS70 Knock-out - Immco Diagnostics] and inmunoblot [ANA+DFS70 Dot Blot - Alphadia]) were performed. Simultaneously, antibodies against extractable nuclear antigens (ENA), nucleosomes (NUS) and histones (HIS) were tested.

Results: In group D, positivity for anti-DFS70 antibodies was confirmed in 7/10 cases, all of them being negative for other ANA. In group M, 2/10 serum samples were positive for anti-DFS70 and 2/10 were positive for anti- NUS antibodies, none of them being positive for anti-ENA. In group C, no sample was positive for anti-DFS70 antibodies, while all of them showed positivity for antibodies against ENA, NUS and HIS.

The detection of anti-DFS70 was found to be equal by the two methods in 8 of the 9 positive cases, being both negative in the others. In no case the presence of anti-DFS70 was associated with a diagnosis of SAD.

Conclusions: Both IIF and immunoblot are suitable methods for detecting anti-DFS70 antibodies. We propose to determine anti-DFS70 and to perform an ENA screening in case of finding a DFS pattern of ANA by IIF, and to investigate anti-DFS70 in other speckled patterns with positive mitosis if no other specificities have been previously found. 